\begin{tabular}{|c|c|c|c|c|c|c|c|c|c|}
\hline \multirow{2}{*}{2} & 0 & $\mathbf{P}$ & 0 & 6 & 3 & \multirow{2}{*}{ B } & \multirow{2}{*}{$\begin{array}{c}\text { Dispatch: } 31.12 .08 \\
\text { Author Received: }\end{array}$} & \multirow{2}{*}{ Journal: OP0 } & CE: Krishna Sarma \\
\hline & Journal & Nam & & Manuscri & t No. & & & & \begin{tabular}{|l|} 
PE: Mahendrakumar \\
\end{tabular} \\
\hline
\end{tabular}

Ophthal. Physiol. Opt. 2008 29: 1-8

\title{
Correlation between the dioptric power, astigmatism and surface shape of the anterior and posterior corneal surfaces
}

\author{
David Mas ${ }^{1}$, Julian Espinosa ${ }^{1}$, Begoña Domenech ${ }^{1}$, Jorge Perez ${ }^{1}$, \\ Henryk Kasprzak ${ }^{2}$ and Carlos Illueca ${ }^{1}$
}

${ }^{1}$ Department of Optica, Universidad de Alicante, c/ Alicante, s.n. 03690, San Vicente del Raspeig, Alicante, Spain, and ${ }^{2}$ Institute of Physics, Wroclaw University of Technology, Wybrzeze

Wyspianskiego 27, Wroclaw, Poland

\begin{abstract}
A knowledge of the shape of the cornea is of major importance for the planning and monitoring of surgery, and for the correct diagnosis of corneal diseases. Many authors have studied the geometry of the second corneal surface in the central region and it has been stated that there is a high correlation between the central radii of curvature and asphericities of the two corneal surfaces. In this work we extend this study to a larger, central, 6-mm diameter of the cornea. Surface height data, obtained with an Oculus Pentacam from 42 eyes from 21 subjects, were analysed to yield surface power vectors. Corneal heights of both surfaces were also decomposed into low-order Zernike polynomials and the correlations between each of the power vectors and low-order Zernike coefficients for the two surfaces were studied. There was not only a strong correlation between spherical powers and Zernike defocus coefficients, but also between the astigmatic components. The correspondence between the astigmatisms in both surfaces found here can be of the utmost importance in planning optical surgery, since perfect spherical ablation of the first surface does not assure total correction of corneal astigmatism.
\end{abstract}

Keywords: cornea, pachymetry, Scheimpflug camera, Zernike polynomials

\section{Introduction}

The principal refracting element and contributor to the aberrations of the human eye is the cornea. Modelling of the refraction in the eye therefore requires an accurate description of the anterior and the posterior corneal surfaces. Some eye models treat the cornea as a single refracting surface which separates air $(n=1)$ from the aqueous humour with an equivalent keratometric index $n_{k}=1.3375$ (Guirao and Artal, 2000). Such a simple approach has several problems. In particular, the typical keratometric index is too high and results in an overestimation of the

Received: 8 September 2008

Revised form: 12 November 2008

Accepted: 12 December 2008

Correspondence and reprint requests to: David Mas.

Tel.: + 349659 03400; Fax: + 34965903464 .

E-mail address: david.mas@ua.es corneal optical power (Dubbelman et al., 2006; Espinosa et al., 2007). Moreover, although the second corneal surface only contributes to a $10 \%$ of the total refractive power of the eye, a precise knowledge of its morphology is needed for the correct diagnosis and monitoring of corneal diseases or surgical interventions.

Several groups have studied the relationship between the anterior and posterior corneal surfaces. Edmund (1994), Garner et al. (1997) and Lam and Douthwaite (2000) found a high correlation between the two surface curvatures. Dubbelman et al. (2002), using Scheimpflug photography, found a clear correlation between anterior and posterior corneal asphericities. Dubbelman et al. (2007) went on to explore the contribution of the posterior surface to the corneal coma aberration. Agerelated changes in corneal biometry were studied by Dubbelman et al. (2007) and Atchison et al. (2008), who also compared the curvature radius and astigmatism of the two corneal surfaces. 
The existence of a strong correlation between the shapes of the two corneal surfaces in healthy eyes is not surprising. In absence of pathologies or external forces, the growth of the corneal tissue should be approximately uniform. Our hypothesis is that, in normal circumstances, the shape of one corneal surface is transferred to the other one and major geometrical features describing the first surface will also be found on the second surface. As a consequence of this, in the presence of astigmatism, both surfaces must be astigmatic and their asymmetry must also be highly correlated. Following this idea, Oshika et al. (1998) evaluated the regular and irregular astigmatism of the posterior surface and correlated these data with those for the anterior surface. They found that there exists a strong correlation between both surfaces and that the nonspherical components of the second surface are not negligible. Lam and Douthwaite (2000) compared the curvature radii of the vertical and horizontal meridians of both corneal surfaces and found a strong correlation between them. Unfortunately, these authors did not explicitly describe the refractive state of their subjects: according to their results we guess that they limited their analysis to normal emmetropic eyes.

It can be seen that several past studies have analyzed the relationship between the corneal surfaces. However, there are two main drawbacks to existing work. First, the majority of studies have been limited to curvature values in the central cornea or to a small number of meridians, so that a significant area of the corneal surface was omitted from the analysis. The second drawback deals with the statistical analysis of the data. Many of the cited works obtained averaged values over all the population under study, thus giving reference values for curvature and astigmatism of the corneal surfaces. We know that the influence of the second surface on the total optical power is low, and its effect on total astigmatism is almost negligible. Moreover, corneal variability regarding astigmatism is large enough to mask the effects of the second surface. These combined circumstances - low average values and large intersubject variability - may mask individual variations and hence fail to yield evidence of shape correspondence between the corneal surface geometries of individual eyes.

In this work we analyze correlations between shape descriptors of the first and second corneal surfaces within a natural pupil size for individual subjects. Measurements were taken with a commercial Schelimpflug camera (Oculus Pentacam) Both anterior and posterior surfaces are reconstructed from corrected data. We have based our analysis on the comparison of spherical and astigmatic descriptors of the surfaces, such as power vector and Zernike coefficients. We show that first- and second-surface coefficients are linearly correlated. This permits modelling of the second surface by direct measurement of the first surface.

\section{Subjects and methods}

\section{Subjects}

Twenty-one adult emmetropic and ametropic subjects without ocular pathology were selected. Contact lens users and patients having undergone ocular surgery were excluded, as well as any irregular corneal astigmatism. Subjects taking part in this study were selected from staff and students of the School of Optics and Optometry of the University of Alicante who met the above selection criteria. We adhered to the tenets of the Declaration of Helsinki during this study. All participants were informed about the nature and purpose of the study and all of them provided informed consent.

\section{Methods}

Corneal measurements were taken with an Oculus Pentacam. The Oculus Pentacam is a Scheimpflug imaging system that allows measurement of 25 meridians in $1 \mathrm{~s}$ and provides a three-dimensional model of the eye's anterior segment. The posterior corneal surface and the anterior crystalline lens surface are calculated by ray tracing, compensating the optical and geometrical distortion of the system itself. Based on this model, the system provides corneal pachymetry and the topography of both corneal surfaces. Shankar et al. (2008) have explored the reliability of this device in measuring the anterior segment. The system allows ASCII files with data from corneal elevation and point-to-point pachymetry to be exported. These were used for the reconstruction of the first and second corneal surfaces. Zernike polynomials for both surfaces were obtained in polar coordinates $(\rho, \theta)$ by using our own software implemented on MATLAB. Sign and normalization follow the convention suggested by the Optical Society of America Standardization Committee (Thibos et al., 2000):

$Z_{n}^{m}(\rho, \theta)=N_{n}^{m} R_{n}^{|m|}(\rho) \cos (m \theta) ;$ if $m \geq 0$
$Z_{n}^{m}(\rho, \theta)=-N_{n}^{m} R_{n}^{|m|}(\rho) \sin (m \theta) ;$ if $m<0$

being $N_{n}^{m}$ a normalization factor and, $R_{n}^{|m|}(\rho)$ the Zernike radial polynomial, both given by:

$R_{n}^{|m|}(\rho)=\sum_{s=0}^{(n-|m|) / 2} \frac{(-1)^{s}(n-s) !}{s ![0.5(n+|m|)-s] ![0.5(n-|m|)-s] !} \rho^{n-2 s}$ 
$N_{n}^{m}=\sqrt{\frac{2(n+1)}{\left(1+\delta_{m 0}\right)}}$

We also obtained and compared the power vectors for the wavefronts emerging from the first and second surfaces (Thibos et al., 2002; Iskander et al., 2007), in particular the spherical and astigmatic components $M$, $J_{0}$ and $J_{45}$ :

$$
\begin{array}{r}
P V^{k}(m)=\frac{1}{r_{p}^{2}} \sum_{n=2}^{k}\left[\sqrt{\left.\frac{n+1}{1+\delta_{m 0}} \frac{(-1)^{\frac{n-2}{2}}\left(\frac{n+2}{2}\right) !}{2^{\frac{\mid m-3}{2}}\left(\frac{n-2}{2}\right) !} c_{n}^{m}\right]}\right. \\
M^{k}=P V^{k}(0)=\frac{1}{r_{p}^{2}}\left(2 \sqrt{3} c_{2}^{0}-6 \sqrt{5} c_{4}^{0}+12 \sqrt{7} c_{6}^{0}+\cdots\right) \\
J_{0}^{k}=P V^{k}(2)=\frac{1}{r_{p}^{2}}\left(\sqrt{6} c_{2}^{2}-3 \sqrt{10} c_{4}^{2}+6 \sqrt{14} c_{6}^{2}+\cdots\right) \\
J_{45}^{k}=P V^{k}(-2)=\frac{1}{r_{p}^{2}}\left(\sqrt{6} c_{2}^{-2}-3 \sqrt{10} c_{4}^{-2}+6 \sqrt{14} c_{6}^{-2}+\cdots\right)
\end{array}
$$

where $c_{m}^{n}$ denotes the Zernike coefficient of radial order $n$ and azimuthal frequency $m$ and $r_{\mathrm{p}}$ is the pupil radius $\delta_{m 0}$ is the Kronecker delta function and $k$ is the maximum order of the polynomical decomposition. In our case we extended the analysis up to $k=7$. Notice that spherical and astigmatic components do not correspond with the typical optometric sphere and cylinder, since they sum all spherical-like and astigmatic-like components. $M, J_{0}$ and $J_{45}$ take into account all the Zernike coefficients of polynomials depending on $\rho^{2}, \rho^{2}$ $\cos (2 \theta)$ and $\rho^{2} \sin (2 \theta)$ respectively. Thus, the power vectors take into account all the lower- and higher order influences over spherical and astigmatism shapes.

We have taken the two corneal surfaces as independent optical elements. We considered a planar wavefront entering each surface and calculated the optical path up to an exit plane, the distance between the input and output planes being the same on both cases. Thus, for the first surface we considered the refraction of a beam passing from air $(n=1)$ to the inner cornea ( $n=1.376)$ and, for the second surface, the refraction between cornea and aqueous humour $(n=1.336)$ (Atchison and Smith, 2005). Thus we could determine the correlation between the powers of the two sides of the cornea and check the compensation effect that the second surface had over the first.

A description based on power vectors allows the correlation in optical power between the surfaces to be explored. Nevertheless, as is evident from Equation (4), the values obtained for both surfaces are affected by higher-order aberrations. This can mask correspondences between the basic surface shapes. Thus, it is also useful to directly compare surface geometries instead of optical powers. To do so we have compared the loworder Zernike coefficients for both surfaces. Since highorder (third-order and above) coefficients only describe small imperfections in the surfaces, it is not reasonable to expect as high a degree of correspondence between the higher-order Zernike coefficients of first and second corneal surface as for the lower-order coefficients. Thus, in addition to power vectors we have extended our analysis to include the defocus $\left(c_{2}^{0}\right)$ and astigmatism coefficients $\left(c_{2}^{-2}\right.$ and $\left.c_{2}^{2}\right)$. Since they are not proper shape descriptors, we have also compared tilt coefficients $\left(c_{1}^{-1}\right.$ and $\left.c_{1}^{1}\right)$ due to the fact that they are very sensitive to misalignments and can provide very useful information about the corneal geometry.

We have used data from the 42 real corneas, 21 from right eyes and 21 from left eyes. We took six different measures from each cornea and used mean data in our calculations. Data were obtained over the central 6-mm diameter of the cornea. Statistics for individual eyes and the whole set were calculated. In all mathematical analyses for both eyes we set the positive direction of our horizontal axis $\left(0^{\circ}\right.$ semi-meridian) as the one pointing nasally.

\section{Results}

The following figures show the correlations between the values of the geometrical parameters describing the first and second corneal surfaces. Each point in the graphs corresponds the average values for each eye, as measured six times. We would like to remark that we are only comparing parameters related to the principal ammetropias, and the reliability of the Pentacam in measuring such parameters has been shown to be good (Shankar et al., 2008). Atchison et al. (2008) have some criticisms of the reliability of Pentacam for determining corneal surface shape. Our own experience suggests that some parameters given by the Pentacam are not accurate, but we did not find any problem with exported data. Thus, we conclude that there are some bugs in the calculation software, but have no reason to doubt the measurements themselves.

In all the figures we distinguish between left and right eyes. In many cases the statistics are very similar in both eyes because of bilateral symmetry. We have summarized results in the figures and captions by giving the regression lines for the whole set (i.e. including both the right and left eyes). In Table 1 we summarize the leastsquares regression line results for the separate right and left eye data and for all eyes .

Figure 1 shows the $M$ vector (see Equation 4 ) for the eyes under study. First we can see that the back surface compensates about $10 \%$ of the refractive power of the front surface. Correspondence between both surfaces is good, with a correlation coefficient $r^{2}=0.74$. 
Table 1. Linear fit values $(y=a x+b)$ for all coefficients studied.

\begin{tabular}{|c|c|c|c|c|c|c|c|c|c|}
\hline & \multicolumn{3}{|c|}{ Left eye } & \multicolumn{3}{|c|}{ Right eye } & \multicolumn{3}{|c|}{ Both eyes } \\
\hline & $a$ & $b$ & $r^{2}$ & $a$ & $b$ & $r^{2}$ & $a$ & $b$ & $r^{2}$ \\
\hline$M$ & -0.12 & 0.01 & 0.70 & -0.13 & 0.10 & 0.79 & -0.13 & 0.06 & 0.74 \\
\hline$J_{0}$ & -0.13 & 0.18 & 0.50 & -0.12 & 0.17 & 0.41 & -0.13 & 0.17 & 0.49 \\
\hline$J_{45}$ & -0.08 & $\underline{0.01}$ & $\underline{0.23}$ & -0.10 & -0.01 & $\underline{0.20}$ & -0.10 & -0.00 & 0.23 \\
\hline$c_{2}^{0}$ & 1.26 & $-\overline{2.85}$ & $\overline{0.90}$ & 1.23 & 0.77 & $\overline{0.87}$ & 1.25 & -1.02 & 0.89 \\
\hline$c_{2}^{-2}$ & 1.11 & 0.21 & 0.43 & 1.39 & 0.98 & 0.66 & 1.27 & 0.58 & 0.52 \\
\hline$c_{1}^{-1}$ & 1.73 & -7.69 & 0.36 & 2.04 & -7.25 & 0.42 & 1.90 & -7.26 & 0.52 \\
\hline$c_{1}^{1}$ & 1.07 & 7.65 & 0.37 & $\underline{0.82}$ & $\underline{8.77}$ & $\underline{0.18}$ & 1.03 & 8.23 & 0.31 \\
\hline${ }^{*} C_{1}^{1}$ & -0.82 & $\underline{4.54}$ & $\underline{0.06}$ & $-\overline{0.68}$ & $\overline{8.88}$ & $\overline{0.03}$ & $\underline{0.32}$ & 7.71 & $\underline{0.01}$ \\
\hline
\end{tabular}

Values for the independent parameter $b$ are given in dioptres $(D)$ for the power vectors and microns ( $\mu$ m) for the Zernike coefficients. The underlined results are not statistically significant ( $p$-value $>0.01)$ while for all the others we have $p<0.01$. The last row with $\left({ }^{*}\right)$ refers to the $x$-tilt coefficients without the outlier points

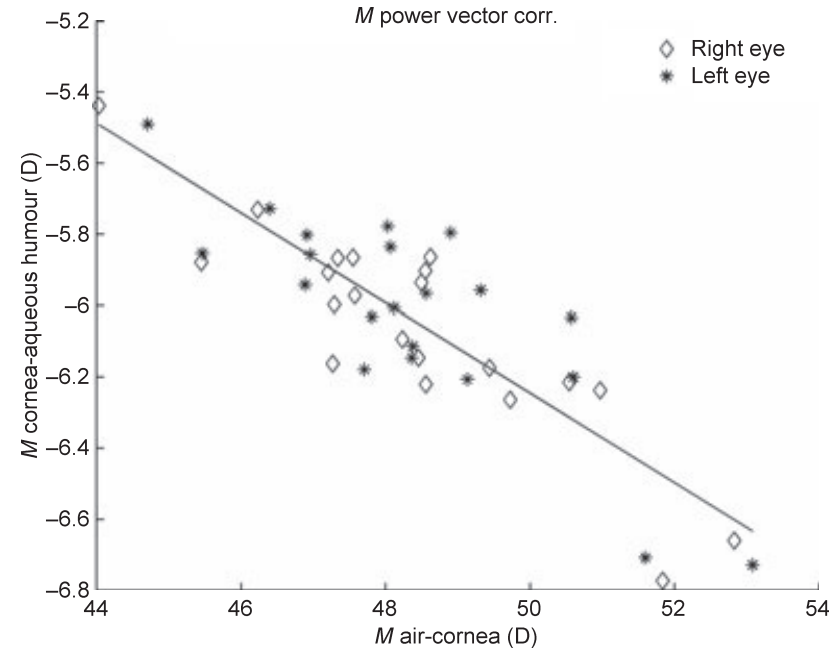

Figure 1. Correspondence between the spherical powers of the first and second corneal surfaces. The regression line is: $y=-0.13 x+$ $0.06 ; r^{2}=0.74 ; p<0.01$.

Figures 2 and 3 show the correlations between front and back corneal surfaces for the $J_{0}$ and $J_{45}$ vectors. These vectors represent the crossed-cylinder surface astigmatisms in the $90 / 180$ and $45 / 135$ axes respectively. Notice that $J_{0}$ component has a good correspondence between the first and the second surface although not as good as that of the sphere component (Figure 1). The $J_{45}$ components, which generally have much smaller magnitudes than their $J_{0}$ counterparts, show poor correlation, the $r^{2}$ coefficient being very low. The $p$ value obtained for left and right eyes $(p>0.05)$ means that correlation is not statistically significant (see Table 1). Although correlation for the whole set is significant $(p<0.01)$, we found that little information can be obtained from this parameter.

As noted earlier, although power vectors in Equation (4) adequately describe the optical performance of the cornea, they do not describe the basic shape of the

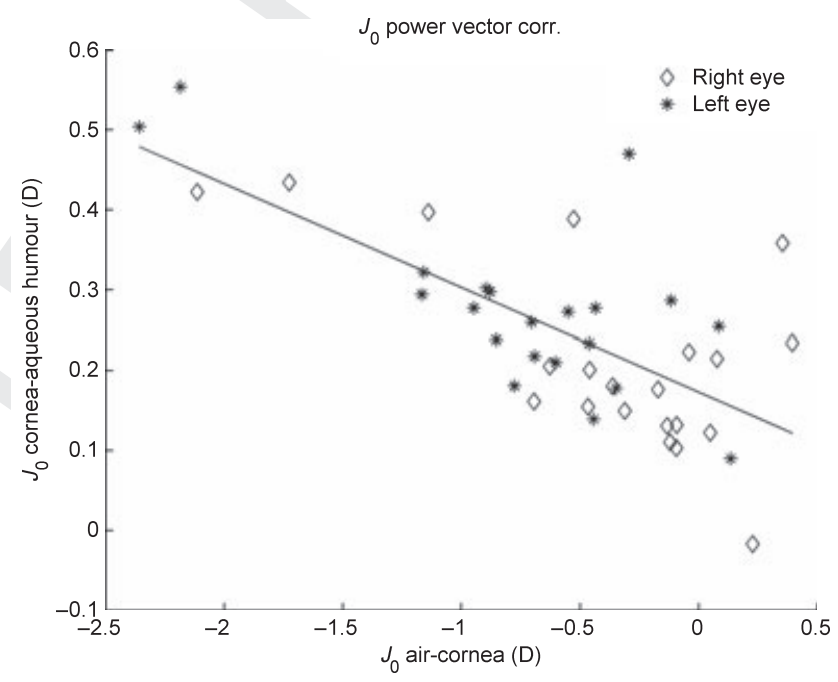

Figure 2. Correspondence between the power vectors $J_{0}$ of the first and second corneal surfaces. The regression line is: $y=-0.13 x+$ $0.17 ; r^{2}=0.49 ; p<0.01$.

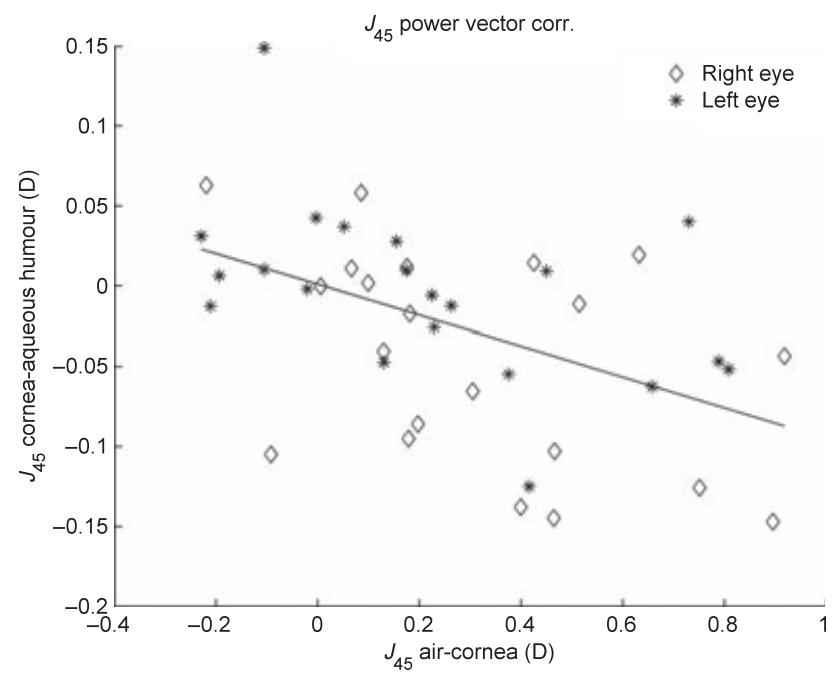

Figure 3. Correspondence between power vectors $J_{45}$ of the first and second corneal surfaces. The regression line is: $y=-0.10 x-$ $0.00 ; r^{2}=0.23 ; p<0.01$. 


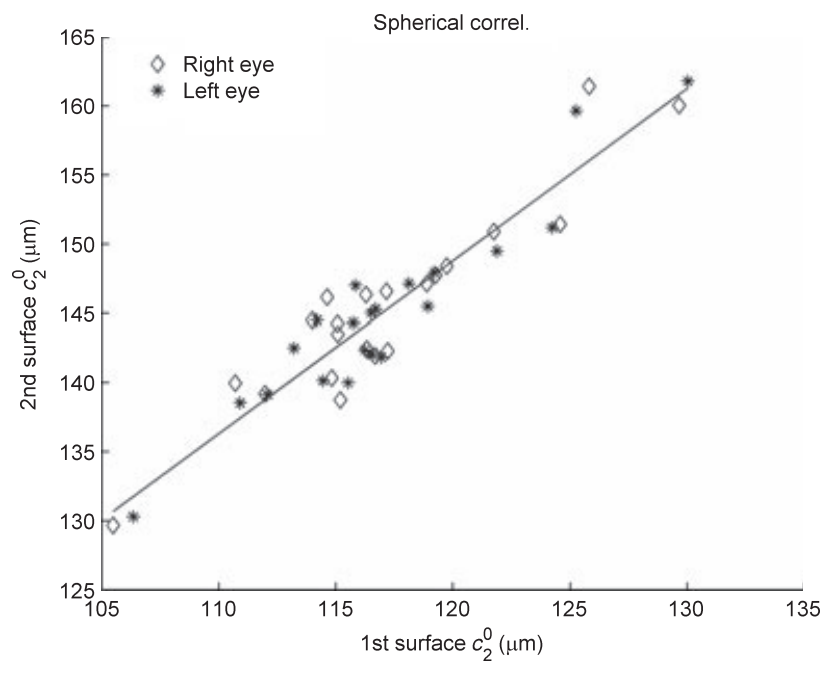

Figure 4. Correspondence between the defocus Zernike coefficients of the first and second corneal surfaces. The regression line is: $y=1.25 x-1.02 ; r^{2}=0.89 ; p<0.01$.

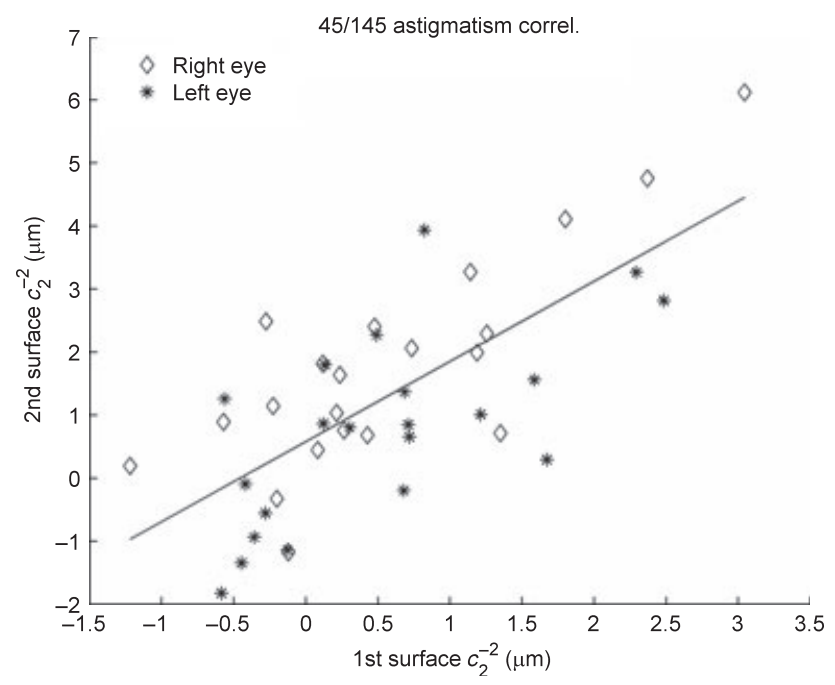

Figure 5. Correspondence between the $45 / 135$ deg astigmatisms of the first and second corneal surfaces. The regression line is: $y=1.27 x+0.58 ; r^{2}=0.52 ; p<0.01$.

surface, so that we have also explored the correlations of the lower-order Zernike coefficients. Figure 4 shows the results for the spherical coefficient $c_{2}^{0}$. We can see there that correlation is very good $\left(r^{2}=0.89\right)$. This coefficient is usually termed 'defocus'. Since we have not removed the reference sphere, it directly represents the spherical component of the surface shape.

Figures 5 and 6 shows the behaviour of the $45 / 135 \mathrm{deg}$ and 90/180 deg astigmatism coefficients, $\left(c_{2}^{-2}\right.$ and $\left.c_{2}^{2}\right)$. Again, we can see a clear correspondence between the two surfaces. Notice that correlation between the Zernike coefficients for the surfaces is much better than that obtained between power vectors, although they must be related. For all eyes, the correlation coefficient

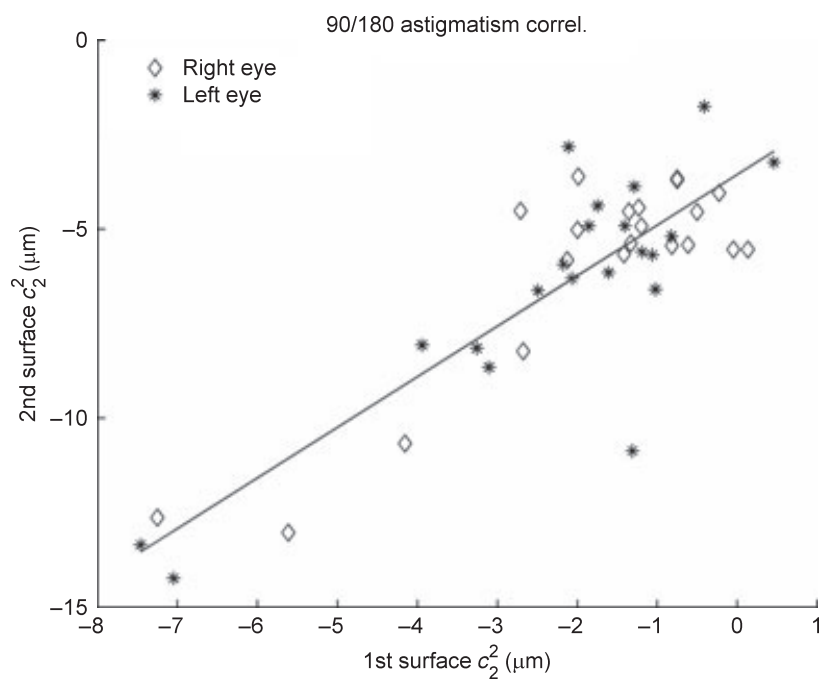

Figure 6. Correspondence between the $90 / 180$ deg astigmatisms of the first and second corneal surfaces. The regression line is: $y=1.34 x-3.55 ; r^{2}=0.72 ; p<0.01$.

for the $90 / 180$ deg-astigmatism is very good $\left(r^{2}=0.73\right)$; this as also the case with the $J_{0}$ vector $\left(r^{2}=0.48\right)$. It is, however, surprising that there is a clear correlation between $45 / 135 \mathrm{deg}$ astigmatism of both surfaces $\left(r^{2}=0.52\right)$, since this effect was not so clear in the analysis of the $J_{45}$ vector $\left(r^{2}=0.23\right)$.

We also have compared tilt coefficients. These coefficients are not properly shape descriptors, but orientation descriptors. Tilt correspondence between the two surfaces may provide information about the alignment between them. Moreover, tilt between optical elements is one of the main factors responsible for coma aberration in the eye (Tabernero et al., 2007) and a proper analysis of this coefficient in the cornea may be useful for correct diagnostic of corneal diseases.

Figures 7 and 8 show the $\mathrm{y}$ and $\mathrm{x}$ tilt coefficients $\left(c_{1}^{-1}\right.$ and $\left.c_{1}^{1}\right)$ for the eyes under study. The correlation between the y-components of the tilt is statistically significant, while that for the $\mathrm{x}$ components is not. In the $x$-tilt figure, there are two outlier points at the lower end of the figure that correspond to the same subject. Table 1 includes the regression data when these outlier points are removed. We can see that, without those data, correlation between both corneal surfaces regarding the $\mathrm{x}$-tilt parameter cannot be established.

\section{Discussion}

Corneal biomechanical stability requires that there exists a clear correspondence between the two corneal surfaces.

When comparing power descriptors in Figures 1-3, we find that the second surface tends to optically compensate the first surface, to diminish the overall 


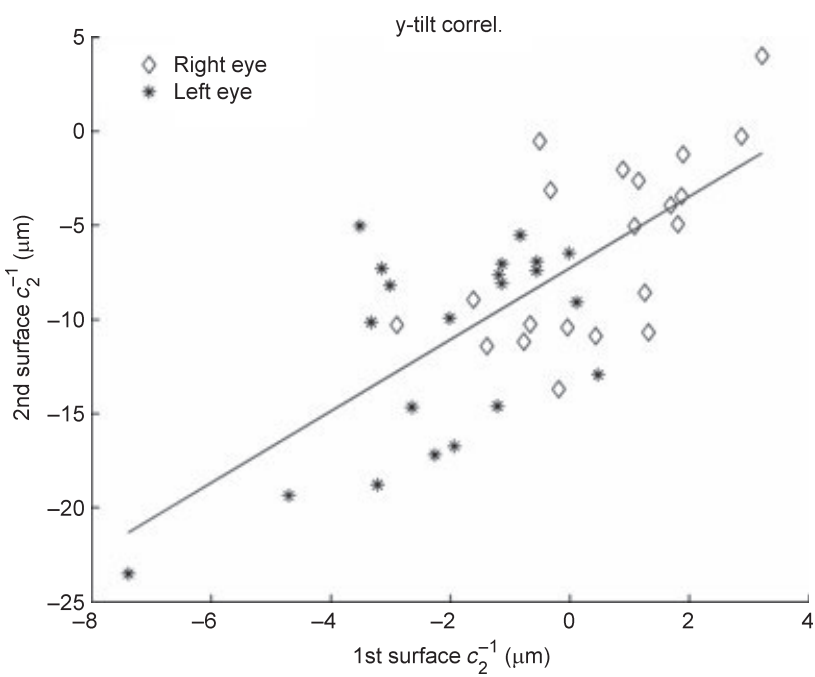

Figure 7. Correspondence between the $y$-tilts of the first and second corneal surfaces. The regression line is: $y=1.90 x-7.26$; $r^{2}=0.52 ; p<0.01$.

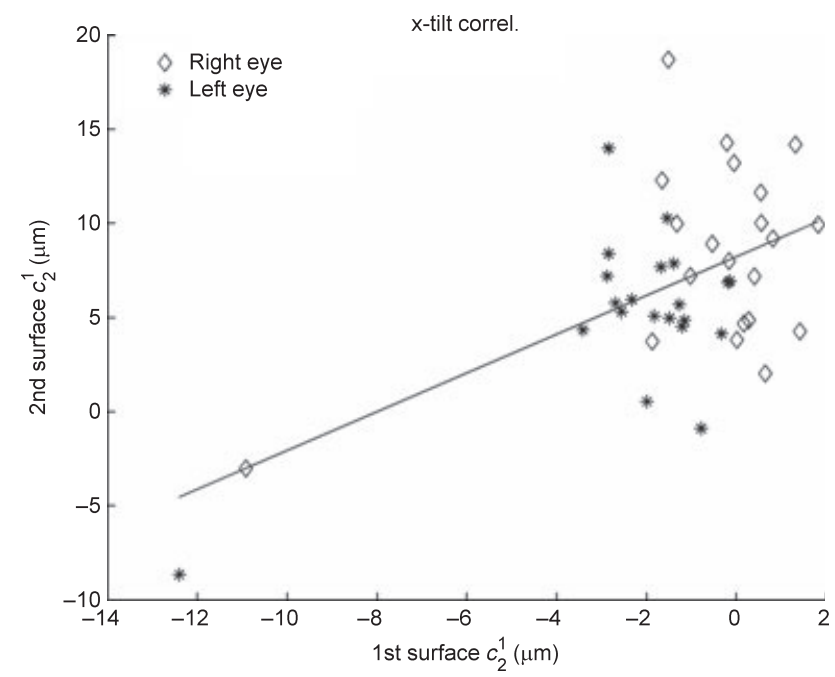

Figure 8. Correspondence between the $x$-tilts of the first and second corneal surfaces. The regression line is: $y=-1.03 x+8.23$; $r^{2}=0.31 ; p<0.01$.

spherical power and astigmatism. Our results for the correspondence between the spherical component agree with those presented by Oshika et al. (1998), who give a ratio of the magnitudes of posterior to anterior spherical power equal to $0.136 \pm 0.006$, compared to our overall value of 0.13 . Our correlation coefficient is higher than theirs, due perhaps to improvement in measuring devices. In order to compare our regular astigmatism with theirs, we have computed the mean values of the first and second surface cylinders. In our case, the ratio of posterior to anterior astigmatism is 0.33 , while Oshika et al. (1998) give an average result of 0.35 . Atchison et al. (2008) give compensation values between the two corneal surfaces for the $J_{0}$ and $J_{45}$ vectors. They state that in emmetropic eyes the second surface compensates $53 \%$ and $23 \%$ of the anterior surface's $J_{0}$ and $J_{45}$ vectors respectively. In our case, we obtain average compensation values of $43 \%$ and $9 \%$. Since our population was not restricted to emmetropic eyes we suggest that our averaged compensation values in the 90/180 deg direction adequately reproduce Atchison et al.'s results. In the oblique case we find that our data dispersion is very large, making our results are unreliable.

In Figures 4-8 we have directly compared the loworder Zernike coefficients for the two corneal surfaces. As we already said, correlations between surface coefficients are better than those obtained for power vectors. We failed to find a good correspondence between the $J_{45}$ components, but a clear correlation appeared in the Zernike $c_{2}^{-2}(45 / 135)$ astigmatism coefficient. This difference might be due to influence of higher-order terms of wavefront decomposition on $J_{45}$ or to the unreliability of the generally small values of $J_{45}$. We would like to remark that we are checking correspondence between coefficients with no distinction over the relative weight of each coefficient in the total amount of aberrations in the eye. High correlation between $c_{2}^{-2}$ astigmatism coefficients but low correlation between $J_{45}$ vectors means that there is no correspondence between higher-order astigmatism in the 45/135 direction. The contrary happens for horizontal/vertical components of the astigmatism and power vectors. Since we are only interested in comparing the basic shape descriptors of the corneal surfaces we will not further analyze the effect of high-aberration coefficients. Having this in mind, from spherical and astigmatism coefficients we can conclude that shapes of front and rear surfaces of the cornea are clearly correlated. Note that slope of the regression line is positive in the three cases (Figures 4-6), confirming that the first surface is always less curved than the second one.

We emphasize that the correlation between the Zernike coefficients, $c_{2}^{-2}$, for oblique astigmatism is, in all cases, worse than that for $90 / 180$ astigmatism, $c_{2}^{2}$, and that the same happens for the corresponding astigmatic power vectors. This is not surprising, since most of astigmatisms are oriented in the vertical and horizontal directions. Notice also that most 90/180 astigmatism coefficients in Figure 6 are negative for both surfaces, which means that the horizontal meridian is flatter than the vertical one. Although the number of subjects is relatively low, this supports the finding that most of the young and middle aged population has with-the-rule corneal astigmatism (Read et al., 2007).

When comparing the tilt coefficients of both corneal surfaces, we found that there is a good correlation for the $y$-tilt but not for the $\mathrm{x}$-tilt. This effect could be due to 
a vertical misalignment between the Pentacam's optical axis and the eye axis. However, the y-tilt of the first surface is distributed around the zero-value, which implies that, as a general rule, the eyes are correctly aligned with the measuring device. It is remarkable that the second surface tilt is negative for all cases except one. This means that the second corneal surface is tilted downwards. This effect may be related to the gravitational forces affecting the IOP. Kasprzak and Pierscionek (2002) propose a model for the gravitational sag of the cornea, and describe a situation like this one affecting both corneal surfaces. We suggest that the first surface tilt is due to small vertical misalignments between the Pentacam and the eye, and that this effect will be highly correlated in the two surfaces. Another effect that only affects the second surface is a permanent tilt due to gravity. The viscoelastic properties of the corneal tissue will explain why this deformation is not transmitted from the second to the first surface.

There is a noticeable difference between the tilt characteristics of the left and right eyes (Figures 7 and 8 ). While first surface y-tilt coefficients for right eyes are distributed between negative and positive values, left eyes tend to have negative coefficients. This effect is more noticeable in the $\mathrm{x}$-tilt values: right eyes values are randomly distributed around zero, but left eyes are biased to the temporal direction. In addition, all second surface $x$-tilt values are positive, which means that, in both eyes, this surface is tilted nasally. Although tilt values are not very high, the bilateral symmetry is broken in almost all the eyes. From Table 1, the reader should note that this effect appears in both astigmatic and tilt Zernike coefficients, although, in some cases, the effect is more noticeable than in others. We do not found any special reason on why the left eye correlations are worse than those for the right eye. One hypothesis is that there could be some postural habit in the subjects that led to these results. It could also be related to the eye dominance. Since target fixation is easier in the dominant than in the non-dominant eye, small fixational inaccuracies in the non-dominant eye may lead to the results here presented. In our most recent measuring campaigns we determined the dominant eye as a part of our measuring protocol. Our preliminary results show that there may be some dominancy effect. More work should be done to clarify all these asymmetry effects.

The results here presented can be of the utmost importance for clinicians who are planning optical surgery. Most corneal ablations assume the one-surface corneal model with an equivalent keratometric index. Thus, apart from central pachymetry measurements, little attention is paid to the second corneal surface. Existence of a strong correlation between the astigmatism of both surfaces means that ablating the anterior cornea will break the equilibrium of the corneal structure. IOP (intraocular pressure) will naturally tend to restablish the optimal mechanical configuration. Thus, astigmatism in the second surface can transfer a residual astigmatism to the first corneal surface just to equilibrate the internal forces. Although the optical power of the second surface is small, its influence over a residual astigmatism must not be neglected.

\section{Acknowledgements}

This work has been partially supported by the Spanish Ministry of Education and Science through the project nr. FIS2005-05053. Julian Espinosa and Jorge Perez acknowledge finnantial support from the University of Alicante through the project GRE07-7P. Henryk Kasprzak acknowledges the University of Alicante its economical support through the project Senior 08/ 07.

\section{References}

Atchison, D. A. and Smith, G. (2005) Chromatic dispersion of the ocular media of human eyes. J. Opt. Soc. Am. A 22, 29-37.

Atchison, D. A., Markwell, E. L., Kasthurirangan, S., Pope, J. M., Smith, G. and Swann, P. G. (2008) Age-related changes in optical and biometric characteristics of emmetropic eyes. J. Vis. 8, 1-20.

Dubbelman, M., Weeber, H. A. and van der Heijde, R. G. L. (2002) Radius and asphericity of the posterior corneal surface determined by corrected Scheimpflug photography. Acta Opththalmol. Scand. 80, 379-383.

Dubbelman, M., Sicam, V. A. D. P. and van der Heijde, R. G. L. (2006) The shape of the anterior and posterior surface of the aging human cornea. Vision Res. 46, 993-1001.

Dubbelman, M., Sicam, V. A. D. P. and van der Heijde, R. G. L. (2007) The contribution of the posterior surface to the coma aberration of the human cornea. J. Vis. 7, 1-8.

Edmund, C. (1994) Posterior corneal curvature and its influence on corneal dioptric power. Acta Ophthalmol. Scand. 72, 715-720.

Espinosa, J., Rouarch, J., Pérez, J., Illueca, C. and Mas, D. (2007) Geometrical approximations for accurate evaluation of refraction in the human cornea. Optik. 118, 209215.

Garner, L. F., Owens, H., Yap, M. K. H., Frith, M. J. and Kinnear, R. F. (1997) Radius of curvature of the posterior surface of the lens. Optom. Vis. Sci. 74, 496-498.

Guirao, A. and Artal, P. (2000) Corneal wave aberration from videokeratography: accuracy and limitations of the procedure. J. Opt. Soc. Am. A 17, 955-965.

Iskander, R., Davis, R. A., Collins, M. J. and Franklin, R. (2007) Objective refraction from monochromatic wavefront aberrations via Zernike power polynomials. Ophthal. Physiol. Opt. 27, 245-255.

Kasprzak, H. and Pierscionek, B. K. (2002) Modelling the gravitational sag of the cornea and the subsequent quality of the refracted image. J. Mod. Opt. 49, 2153-2166. 
Lam, A. K. C. and Douthwaite, W. A. (2000) The ageing effect on the central posterior corneal radius. Ophthal. Physiol. Opt. 20, 63-69.

Oshika, T., Tomidokoro, A. and Tsuji, H. (1998) Regular and irregular refractive powers in the front and back surfaces of the cornea. Exp. Eye Res. 67, 443-447.

Read, S. A., Collins, M. J. and Carney, L. G. (2007) A review of astigmatism and its possible genesis. Clin. Exp. Optom. 90, 5-19.

Shankar, H., Taranah, D., Chandramalar, F., Santhirathelagan, T. and Pseudovs, K. (2008) Anterior segment biometry with the Pentacam: comprehensive assesment of repeatabil- ity of automated measurements. J. Cataract Refract. Surg. 34, 103-113.

Tabernero, J., Benito, A., Alcón, E. and Artal, P. (2007) Mechanism of compensation of aberrations in the human eye. J. Opt. Soc. Am. A 24, 3274-3283.

Thibos, L. N., Applegate, R. A., Schwiegerling, J. T. and Webb, R. H. (2000) Standards for reporting the optical aberrations of eyes. Trends Opt. Photon 35, 110-130.

Thibos, L. N., Hong, X., Bradley, A. and Cheng, X. (2002) Statistical variation of aberration structure and image quality in a normal population of healthy eyes. J. Opt. Soc. Am. A 19, 2329-2348. 


\section{Author Query Form}

\section{Journal: $\quad$ OPO}

Article: $\quad 632$

Dear Author,

During the copy-editing of your paper, the following queries arose. Please respond to these by marking up your proofs with the necessary changes/additions. Please write your answers on the query sheet if there is insufficient space on the page proofs. Please write clearly and follow the conventions shown on the attached corrections sheet. If returning the proof by fax do not write too close to the paper's edge. Please remember that illegible mark-ups may delay publication.

Many thanks for your assistance.

\begin{tabular}{|l|l|l|}
\hline Query reference & Query & Remarks \\
\hline 1 & $\begin{array}{l}\text { AUTHOR: Please give manufacturer information for Scheimpflug camera: } \\
\text { town, state (if USA), and country. }\end{array}$ & \\
\hline
\end{tabular}




\section{Please correct and return this set}

Please use the proof correction marks shown below for all alterations and corrections. If you wish to return your proof by fax you should ensure that all amendments are written clearly in dark ink and are made well within the page margins.

\begin{tabular}{|c|c|c|}
\hline Instruction to printer & Textual mark & Marginal mark \\
\hline Leave unchanged & ... under matter to remain & ( ) \\
\hline $\begin{array}{l}\text { Insert in text the matter } \\
\text { indicated in the margin }\end{array}$ & $h$ & $\begin{array}{l}\text { New matter followed by } \\
h \text { or } h \otimes\end{array}$ \\
\hline Delete & $\begin{array}{l}\text { I through single character, rule or underline } \\
\text { or }\end{array}$ & $\sigma$ or $\sigma(x)$ \\
\hline $\begin{array}{l}\text { Substitute character or } \\
\text { substitute part of one or } \\
\text { more word(s) }\end{array}$ & I through letter or & $\begin{array}{l}\text { new character / or } \\
\text { new characters / }\end{array}$ \\
\hline Change to italics & — under matter to be changed & $\leftarrow$ \\
\hline Change to capitals & $\equiv$ under matter to be changed & $\equiv$ \\
\hline Change to small capitals & $=$ under matter to be changed & $=$ \\
\hline Change to bold type & $\sim$ under matter to be changed & $\sim$ \\
\hline Change to bold italic & $\bar{\sim}$ under matter to be changed & $\underline{s i n}$ \\
\hline Change to lower case & Encircle matter to be changed & $\Rightarrow$ \\
\hline Change italic to upright type & (As above) & \\
\hline Change bold to non-bold type & (As above) & \\
\hline Insert 'superior' character & $\begin{array}{l}/ \text { through character or } \\
K \text { where required }\end{array}$ & $\begin{array}{l}y^{\prime} \text { or } y \\
\text { under character } \\
\text { e.g. } y^{2} \text { or } y^{2}\end{array}$ \\
\hline Insert 'inferior' character & (As above) & $\begin{array}{l}\lambda \\
\text { over character } \\
\text { e.g. } \hat{\Sigma}\end{array}$ \\
\hline Insert full stop & (As above) & $\odot$ \\
\hline Insert comma & (As above) & , \\
\hline Insert single quotation marks & (As above) & $\begin{array}{l}\dot{y} \text { or } \dot{x} \text { and/or } \\
\dot{y} \text { or } \dot{y}\end{array}$ \\
\hline Insert double quotation marks & (As above) & $\begin{array}{l}\ddot{y} \text { or } \ddot{y} \text { and/or } \\
\ddot{y} \text { or } \ddot{y}\end{array}$ \\
\hline Insert hyphen & (As above) & 1 \\
\hline Start new paragraph & 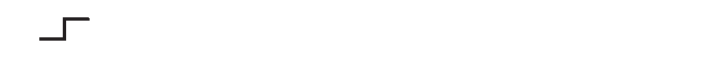 & 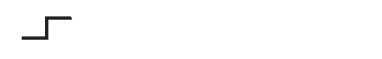 \\
\hline No new paragraph & $\infty$ & $\omega$ \\
\hline Transpose & $\sqcup$ & $\sqcup$ \\
\hline Close up & linking $\bigcirc$ characters & \\
\hline $\begin{array}{l}\text { Insert or substitute space } \\
\text { between characters or words }\end{array}$ & $\begin{array}{l}\text { I through character or } \\
\Lambda \text { where required }\end{array}$ & \\
\hline $\begin{array}{l}\text { Reduce space between } \\
\text { characters or words }\end{array}$ & $\begin{array}{l}\text { between characters or } \\
\text { words affected }\end{array}$ & $\uparrow$ \\
\hline
\end{tabular}

